\title{
Variables de deterioro en la gestión de la sobremesa cubana y la protección al consumidor
}

DOI: https://doi.org/10.33262/ap.v2i1.21

\begin{abstract}
(c) (i) (8)(2)
Variables of deterioration in the management of the Cuban desktop and consumer protection
\end{abstract}

Pedro Pablo Milán Fonseca., ${ }^{1}$ Olivia González Basanta., ${ }^{2}$ \& Patricia Guerra Rodríguez. ${ }^{3}$

\begin{abstract}
.
The deficient management of Cuban tabletop products in Havana, which deteriorates the consumer protection system in catering establishments for tourism, was the main problem of this research. The identification of the variables that deteriorate the consumer protection system in such management, contributes to an improvement in food and beverage services, favors the marketing of national products and customer satisfaction. In order to strengthen the consumer protection system, in accordance with international standards, the research was structured in three phases, corresponding to: (1) consulting secondary sources of information, through documentary analysis and a referential search on the Internet; (2) consulting primary sources of information, using measurement, descriptive statistics, interviews, surveys and scientific observation; (3) the analysis, synthesis and resolution, starting from a statistical design for the elaboration of tables, graphs, histograms, dendograms, classification trees and hierarchical conglomerates, and using Pareto and Ishikawa diagrams. The main result of the investigation was the identification of said variables and criteria, which served as the basis for the development of a procedure that mitigates the negative impact they have on the consumer protection system.
\end{abstract}

\footnotetext{
${ }^{1}$ Universidad de La Habana. Facultad de Turismo La Habana, Cuba. ppmilan40@ gmail.com

${ }^{2}$ Universidad de La Habana. Facultad de Turismo La Habana, Cuba. glez.basanta@gmail.com

${ }^{3}$ Universidad de La Habana. Facultad de Turismo La Habana, Cuba. patryr99@gmail.com
} 
Keywords: Cuban after-dinner, Consumer protection, Tourism, Restaurants.

\section{Resumen.}

La deficiente gestión de los productos de sobremesa cubana en La Habana, que deteriora el sistema de protección al consumidor en entidades de restauración para el turismo, constituyó la principal problemática de la presente investigación. La identificación de las variables que deterioran el sistema de protección al consumidor en dicha gestión, contribuye a una mejora en los servicios de alimentos y bebidas, favorece la comercialización de productos nacionales y la satisfacción del cliente. Con el fin de fortalecer el sistema de protección al consumidor, atendiendo a estándares internacionales, la investigación se estructuró en tres fases, correspondientes a: (1) la consulta a fuentes secundarias de información, a través del análisis documental y la búsqueda referencial en Internet; (2) la consulta a fuentes primarias de información, empleando la medición, estadística descriptiva, entrevistas, encuestas y observación científica; (3) el análisis, síntesis y resolución, partiendo de un diseño estadístico para la elaboración de tablas, gráficos, histogramas, dendogramas, árboles de clasificación y conglomerados jerárquicos, y empleando los diagramas de Pareto e Ishikawa. La investigación arrojó como resultado principal la identificación de dichas variables y criterios, los cuales sirvieron como fundamento para la elaboración de un procedimiento que atenúe el impacto negativo que ejercen sobre el sistema de protección del consumidor.

Palabras claves: Productos de sobremesa cubana, protección al consumidor, Turismo, restauración.

\section{Introducción.}

En la contemporaneidad, cada vez más globalizada y convulsa, uno de los sectores de más dinámico crecimiento es el turismo. El hecho de que el 10\% del PIB mundial (por efecto directo, indirecto o inducido) corresponda al sector turístico (OMT, 2020) justifica que cada vez más naciones, incluso las más industrializadas, apuesten muy seriamente por el turismo como sector estratégico de crecimiento económico.

El sector turístico se ha convertido en una herramienta fundamental para el desarrollo económico, social y cultural de los territorios, dado su elevado aporte a la creación de empleo y riqueza; su carácter equilibrador de la balanza de pagos; su aporte al desarrollo armónico y equilibrado del territorio, afectando positivamente a las zonas más desfavorecidas; así como su naturaleza generadora de efectos dinamizadores en otros sectores económicos.

Partiendo de esta premisa, hoy el sector se sustenta sobre un modelo basado en criterios de calidad, competitividad, diversificación y sostenibilidad, principios que tratan de responder a las demandas del turista actual. Este modelo, y los principios inherentes al mismo, se 
enfrentan a un problema que se ha ido extendiendo progresivamente: el intrusismo en la prestación de servicios turísticos, específicamente, la prestación de actividades turísticas por establecimientos y agentes no cualificados para desarrollarla, que proporcionan sus servicios sin estar habilitados para ello y, por tanto, sin ajustarse a los criterios de calidad, seguridad e infraestructura que exige el ordenamiento jurídico turístico. Este fenómeno entorpece las bases estructurales del modelo turístico vigente.

La existencia de intrusismo profesional en el sector afecta también a la definición y configuración de la política pública de turismo. La evaluación de las necesidades de la oferta se ve desvirtuada y, por tanto, la ordenación y regulación de la actividad, la elaboración de programas de comercialización y planes de promoción, así como el establecimiento de ayudas y subvenciones, no responden a las necesidades reales del sector, reduciendo así la eficacia de la actuación de la administración turística.

Ante esta situación entendida como reto para el propio funcionamiento del sector, la administración turística está poniendo en marcha diversas actuaciones cuya finalidad es frenar y erradicar el fenómeno del intrusismo profesional en el sector, si bien el análisis del tratamiento de este problema debe partir de una serie de premisas básicas que sirvan como punto de reflexión.

Como uno de los renglones propios del desarrollo turístico se encuentra el gastronómico, partiendo del papel de los productos y tradiciones culinarias cubanas. La gastronomía es inclusiva del espacio de la sobremesa, momento donde los comensales buscan formas de resaltar la experiencia gustativa combinando infusiones, espirituosos, vinos, entre otros productos que, en el caso autóctono cubano, manifiesta la inclusión de los habanos como elemento integrador.

En tal sentido, todo un conjunto de actividades que se han venido desarrollando en Cuba en los últimos años -como cursos de formación de Capitán de Salón, Sommeliers, La sobremesa en la Restauración, impartidos en las escuelas de hostelería y turismo del país, creación de aulas especializadas de sommeliería en estas mismas escuelas, concursos nacionales e internacionales de sommeliería, creación de los clubes de sommelier en las distintas provincias del país y los Festivales Internacionales del Habano en Cuba (EAEHT, 2016)-, han ido dando pasos sustanciales en cuanto a la cultura y conocimiento del servicio de sobremesa para el personal que trabaja en el turismo y sectores afines, lo que aporta, como elemento clave, la distinción que ofrece este servicio para las instalaciones tanto hoteleras como extrahoteleras.

Aun así, en instalaciones de restauración hoteleras y extra hoteleras de La Habana, se aprecian dificultades en cuanto a la gestión del producto servicio de sobremesa, lo que reduce los resultados positivos de dichas instalaciones, así como la calidad del servicio prestado a los consumidores. Expreso en causas internas como la afectación a la satisfacción del cliente, 
la presencia de recursos humanos poco capacitados y deficiencias en la comercialización por la insuficiente gestión de marketing; condicionadas en externas como problemas en la economía nacional debido a la deficiente relación entre las formas de la economía, el deficiente empleo de las Nuevas Tecnologías de la Información y las Comunicaciones, presente en la ausencia del comercio electrónico en la gestión y la no existencia de normativas y procedimientos establecidos referentes a la gestión de este servicio, que le permitan tomar decisiones para la mejora del mismo, todo esto deteriorando el sistema de protección al consumidor propio de servicios de restauración turísticos.

Identificar las variables que deterioran el sistema de protección al consumidor en la gestión de productos de sobremesa cubana en instalaciones que presten servicios al turismo en La Habana, se plantea como objetivo principal de la investigación.

\section{Revisión bibliográfica.}

Las Directrices de Naciones Unidas para la Protección del Consumidor (UNCTAD, 2016), establecen en su apartado D las Normas para la seguridad y calidad de los servicios y bienes de consumo, donde se hace énfasis en la pertinencia de personal cualificado en la prestación de servicios globales a consumidores.

Como se formula,

33. Los Estados Miembros deben, según proceda, formular o promover la formulación y aplicación, en los planos nacional e internacional, de normas, voluntarias o de otra índole, de seguridad y calidad de los bienes y servicios, y dar a dichas normas la publicidad apropiada. Las normas y reglamentaciones nacionales relativas a la seguridad y calidad de los productos deben examinarse de tanto en tanto para cerciorarse de que, en lo posible, se ajusten a normas internacionales de aceptación general.

Se hace referencia, además, a normativas especiales de protección a consumidores foráneos a los Estados en cuestión, donde el papel de los servicios turísticos es prioritario de regulación por las Administraciones competentes debido al estado de indefensión inherente a viajeros que visitan otros destinos.

La restauración ha evolucionado enormemente, pasando de pequeños negocios familiares a formar medianas y grandes empresas, lo que conlleva una complejización de sus niveles de gestión (Morcillo, 2012).

La gestión moderna de la restauración aumenta cada vez más su eficacia para no quedarse al margen de las tendencias actuales del mercado. Las funciones de las comidas también han evolucionado y es preciso invertir en tecnología, materiales, preparación del personal, cualquiera que sea la categoría del restaurante, para cumplir las múltiples exigencias del cliente de hoy. 
Muchos de los problemas que se presentan en la restauración moderna tienen sus raíces en la falta de organización, planeación, comercialización y visión hacia el cliente, lo cual conduce a una administración inadecuada y violación de las políticas de protección al consumidor.

Las empresas de restauración son consideradas como empresas de servicio, transformadoras de productos y comercializadoras. La actividad de servicio en este tipo de empresa es esencial; si no se realiza correctamente, los resultados de los factores como producción y comercialización habrán resultado inútiles.

El servicio de cena transita por varias etapas importantes: la primera, sin duda alguna, es la de los aperitivos o entrantes, conformada por frutas frescas, ensaladas de pastas o vegetales; le sigue la presentación de los platos principales, la cual exige, por lo general, el acompañamiento inseparable, y con un perfecto maridaje, de los vinos; posteriormente continúan los postres, que para muchos comensales significan la clausura de la cena. El desconocimiento los limita al privilegio de un cierre maravilloso y por todo lo alto de una cena inolvidable: para el final brillante de una cena se hace imprescindible disfrutar de un majestuoso servicio de sobremesa el cual tiene como protagonistas los espirituosos, las infusiones y los habanos.

El servicio de sobremesa le añade un realce incalculable al final de la cena, dependiendo del nivel de capacitación del sommelier encargado del mismo, que sea capaz de atrapar en el cliente sensaciones nunca antes experimentadas, a través de un magistral ritual del habano y un alto nivel de conocimiento de los espirituosos e infusiones, de manera tal que cautive la atención del cliente y sirva como gancho para hacer de este un repitente permanente.

\section{Productos del servicio de sobremesa cubana.}

\section{El ron cubano.}

Según Fernando Fernández en Cubaron S.A. (2016), el ron es una bebida alcohólica que se obtiene a partir de la caña de azúcar por fermentación, destilación y envejecimiento, generalmente en barricas de roble. La caña de azúcar de origen surasiático y su proceso de destilación de alcoholes con alambique, llegaron a Cuba durante la colonización española. Cuando los ingleses y franceses se instalan en Las Antillas, toman el cultivo de la caña y comienzan a destilar el ron. El primero se desarrolló en Barbados, y aunque el mundo antillano extendió sus dimensiones, la isla que primero elevó el ron a su condición de bebida clásica fue Cuba. Lo que hoy se conoce como ron cubano es el tercer gran estilo en orden de aparición. Varias marcas se impusieron el mundo, entre ellas, Matusalén, Jiquí, Bocoy, Campeón, Obispo, Castillo, Bacardí y Habana Club.

\section{Las infusiones.}

Según RAE (2019), las infusiones son bebidas que se obtienen de diversos frutos o hierbas aromáticas, como té, café, manzanilla, etc, introduciéndolos en agua hirviendo. Este producto 
tiene un origen enigmático, no resuelto todavía por la ciencia ni los historiadores, se desconoce el momento en que empezó a ser usado como brebaje. Aunque recientes investigaciones informan haber descubierto té en la tumba de un emperador chino con al menos 2150 años de antigüedad (AETi, 2016).

La mayoría de los estudiosos coinciden en que la planta era conocida y utilizada en épocas remotas, incluso sus hojas eran mascadas, y en que se pudo haber dejado fermentar el fruto en agua, y beber entonces el jugo resultante. Las infusiones más comunes son: el café, el té, la hierbabuena, la menta, la manzanilla, la tila, el mate y la horchata.

\section{El café cubano.}

La planta del cafeto arribó a Cuba desde República Dominicana en el siglo XVIII, con Don José Gelabert, quien fundó en el Wajay, el primer cafetal de la isla hacia 1748. La avalancha de haciendas cafetaleras se produjo en Cuba desde que la migración franco-haitiana, conocedores de su cultivo, encontraron condiciones idóneas en cuanto a terreno y clima en la Sierra Maestra, a partir de ahí se desarrollaron haciendas cafetaleras que devinieron importantes centros productores y llevaron a Cuba a ser el primer exportador mundial a inicios del siglo XIX (AHS, 2018). Aunque posteriormente el país perdió liderazgo como exportador, mantuvo una alta calidad en el cultivo y beneficio del grano, trasladando esta tradición, como un ritual que pasó de generación en generación, hasta la actualidad.

\section{Los Habanos.}

Fue en Cuba en 1492 cuando la expedición española comandada por Colon vio por primera vez el tabaco en el Nuevo Mundo. Los indios taínos enrollaban y prendían unas hojas misteriosas que llamaban "Cohíba", en una ceremonia desconocida hasta ese momento para los descubridores. Posteriormente el tabaco fue comerciado y plantado por todo el planeta. Sin embrago, el Tabaco Negro Cubano fue considerado el mejor del mundo por las condiciones únicas que ostentan ciertas partes de la Isla para su cultivo (Consejo Regulador de la DOP, 2017). La esencia de esta diferencia parte de cuatro factores: suelos, clima, la variedad del Tabaco Negro Cubano y el saber hacer de los vegueros y torcedores. Otro rasgo distintivo es la definición del término "Habanos", pues todos los Habanos son cubanos, pero no todos los puros cubanos son de esta tipología, se elaboran totalmente a mano, aplicando métodos utilizados por primera vez en La Habana hace siglos, y que perduran invariables casi hasta nuestros días.

\section{Material y métodos.}

La investigación es de tipo descriptiva, se trabajó sobre realidades de la situación actual del objeto de estudio. En el desarrollo de la misma, se hizo necesario el empleo de diferentes métodos teóricos y empíricos, los cuales proporcionaron objetividad y solidez. Entendidos 
los procesos fundamentales a analizar, sintetizar y resolver, se estructuró en tres fases el recorrido investigativo:

\section{Fase 1: Consulta a fuentes secundarias de información}

Se desarrolló una detallada revisión bibliográfica de las fuentes de información existentes, logrando así una mejor comprensión del fenómeno a partir de la aplicación de métodos teóricos como el Histórico-Lógico, el Analítico-Sintético, la Inducción-Deducción, y de métodos empíricos como el análisis documental y la búsqueda referencial en Internet, específicamente para la identificación de las características de la actividad de sobremesa y las pautas del sistema de protección al consumidor a nivel internacional, aportada por otros investigadores. Estos criterios arrojados en la revisión, internacionalmente reconocidos, sirvieron de base para la presente investigación.

\section{Fase 2: Consulta a fuentes primarias de información}

El desarrollo de esta fase estuvo basado, principalmente, en el desarrollo y aplicación de métodos, técnicas y herramientas que permitieron la obtención de información primaria.

Del método estadístico-matemático se empleó la medición, que permitió discernir sobre la esencia del problema, aplicando procedimientos estadísticos que permiten revelar las tendencias, regularidades y las relaciones en el proceso o fenómeno objeto de estudio. En este caso, se utilizó la estadística descriptiva para organizar y clasificar los indicadores cuantitativos obtenidos en la medición.

Se realizaron entrevistas a doce especialistas del tema, procedentes de la Universidad de La Habana, la Escuela Ramal del MINTUR y otras entidades que prestan servicios al turismo, a fin de indagar sobre las tradiciones y el valor cultural de la sobremesa cubana, así como de los productos que la componen -ron, tabaco y café-. Otro tema relevante fue el valor que añade el maridaje a la consecución de la oferta de sobremesa cubana y del producto en sí, incluyendo el cómo se comporta la difusión del mismo. Estas fueron de tipo personal semiestructuradas y compuestas por preguntas abiertas.

Por otra parte, se aplicó una encuesta a consumidores a través de un cuestionario del tipo estructurado, con una selección intencionada de una muestra de 104 consumidores del servicio en cuestión. Esto permitió dimensionar el estado del problema desde diversas perspectivas y grupos de análisis.

La observación científica también se empleó como método eficaz para conocer y tener un acercamiento a la realidad, permitiendo valorar el estado actual de la gestión de ofertas de sobremesa cubana en instalaciones pertenecientes al MINTUR -Palacio de la Artesanía, El Restaurante Bar 1830 y el Complejo "La Cecilia"-. Para ello se aplicó una guía de observación participativa y no participativa. 


\section{Fase 3: Análisis, síntesis y resolución}

En esta fase se procedió a organizar y procesar los datos obtenidos de las fuentes de recopilación de información, así como a la realización de un diseño estadístico para el procesamiento de los datos del cuestionario y su análisis en la elaboración de las variables que interactúan con el sistema de protección al consumidor en la gestión de servicio de sobremesa cubana. Sirvió de apoyo la elaboración de tablas, gráficos, histogramas, dendogramas, árboles de clasificación y conglomerados jerárquicos, utilizando para ello el programa SPSS Statistics versión 22 de IBM. Esto permitió determinar las características del sistema y sus variables de interacción con la gestión de la actividad de sobremesa, así como establecer un marco propositivo para la acción y control de la actividad.

Se empleó el Diagrama de Pareto partiendo de que el Análisis de Pareto es una técnica que separa los "pocos vitales" de los "muchos triviales", utilizada para separar gráficamente los aspectos significativos del problema desde los triviales, de manera que el equipo sepa dónde dirigir sus esfuerzos para mejorar. La herramienta permitió la determinación de la causa principal durante un esfuerzo de resolución del problema. Asimismo, se utilizó un Diagrama de Ishikawa para lograr una representación gráfica de las causas y sub-causas del problema, logrando identificar visualmente la raíz o causa principal, así como las interrelaciones entre el efecto y sus posibles causas, clasificar y relacionar las interacciones entre factores que están afectando al resultado.

\section{Resultados y discusión.}

Las técnicas y herramientas aplicadas arrojaron los siguientes resultados, partiendo de la observación directa, las entrevistas y encuestas aplicadas en la investigación. Se presenta un Diagrama de Pareto, un Diagrama de Ishikawa y la Distribución de Criterios que permiten analizar la gestión del producto de sobremesa cubana.

Figura 1: Diagrama de Pareto

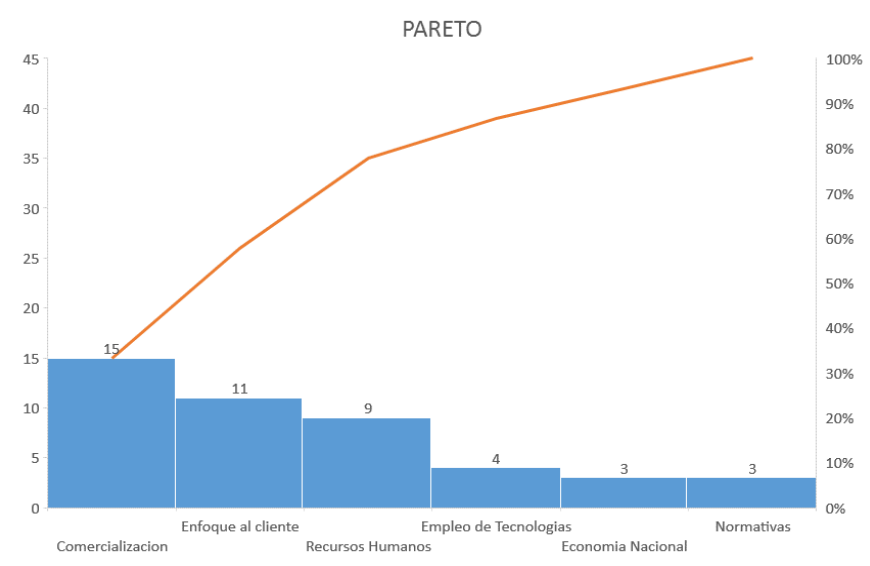

Fuente: Elaboración propia. 
La figura 1 muestra los criterios de los especialistas entrevistados con respecto a las variables que deterioran el sistema de protección al consumidor en entidades donde se ofrece el servicio de sobremesa cubana: los recursos humanos, el enfoque al cliente, las normativas relacionadas, la comercialización de la oferta, la economía nacional y el empleo de tecnologías.

Es un hecho que el habano, el ron y el café cubanos son productos de alto prestigio internacional, exponentes de la cultura cubana. Si bien existe una profunda comercialización de estos tres elementos de manera independiente, no ha sido así para su interrelación como producto de sobremesa en el maridaje cubano. Por otro lado, la investigación demostró que estos productos se ofrecen con poca o ninguna personalización, lo que impide que los viajeros sean capaces de disfrutar una experiencia relacionada con ellos, más allá de su consumo.

Finalmente, los recursos humanos que brindan los servicios de sobremesa no están adecuadamente calificados como Habanosommeliers, especialistas en el maridaje de habanos, ron y café. Estos elementos influyeron en la decisión de los especialistas de posicionar como variables que más contribuyen al deterioro a la Comercialización, seguida del Enfoque al Cliente y los Recursos Humanos.

Figura 2: Diagrama de Ishikawa

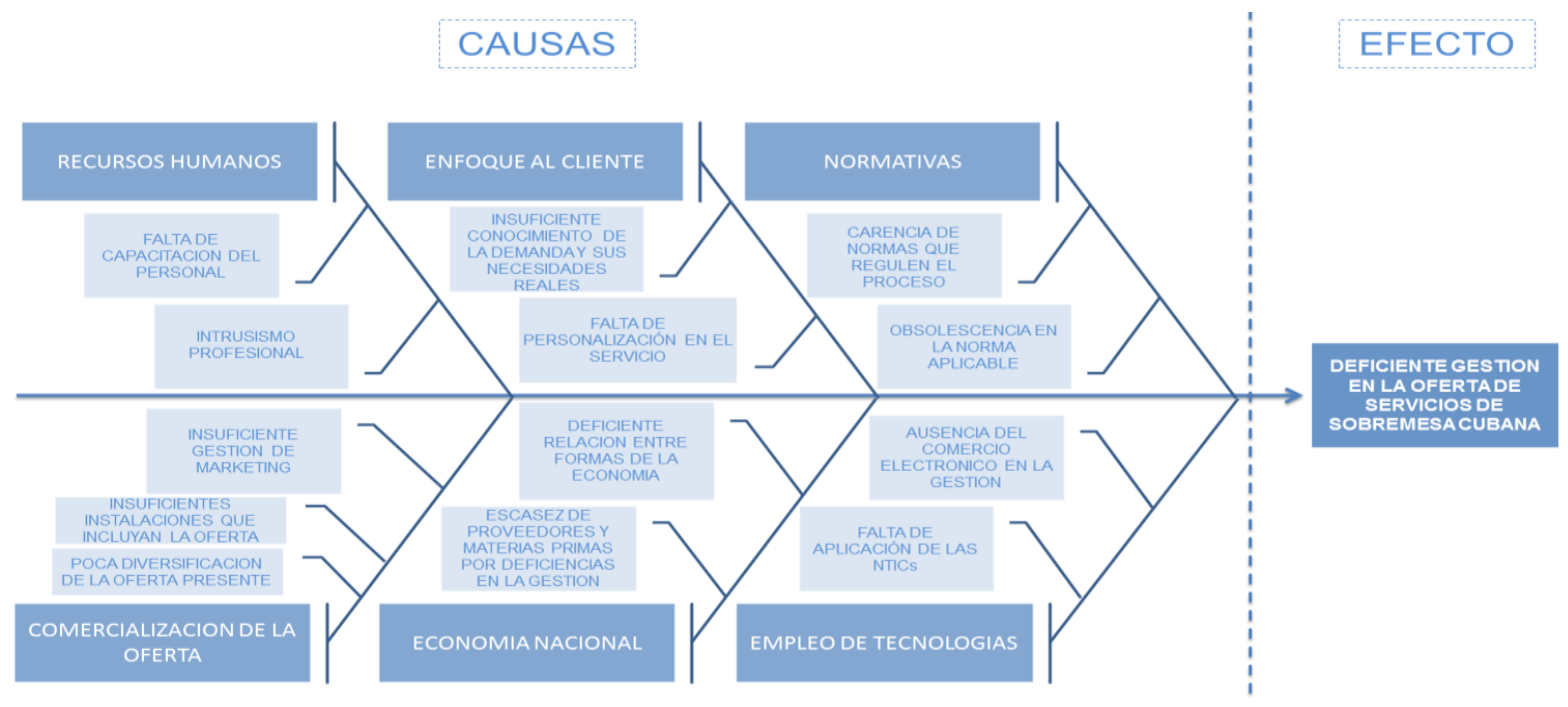

Fuente: Elaboración propia.

Es apreciables que las causas que originan el efecto que se considera el problema fundamental en la investigación -Deficiente gestión en la oferta de servicios de sobremesa cubana-, coinciden con los criterios de los especialistas. El Diagrama de la figura 2 permitió visualizar las sub-causas de cada causa que influyen en el deterioro. 
Se percibe que existe una insuficiente gestión de marketing, pocas instalaciones que ofrecen servicios de maridaje cubano e insuficiente oferta, lo que afecta la comercialización de la misma. A su vez, es insuficiente el conocimiento de la demanda y sus necesidades reales y se muestra una falta de personalización en el servicio, perjudicando el enfoque al cliente. Por último, es perceptible la falta de capacitación del personal, así como el intrusismo profesional a la hora de brindar la oferta de sobremesa cubana.

Tabla 1. Distribución de criterios

\begin{tabular}{|c|c|c|}
\hline Macro & Criterios & $\mathbf{F A}^{\mathrm{a}}$ \\
\hline \multirow{3}{*}{ Recursos Humanos } & Falta de Capacitación del Personal & 5 \\
\hline & Intrusismo Profesional & 3 \\
\hline & Migración a otras actividades & 1 \\
\hline \multirow{3}{*}{ Enfoque al cliente } & Insuficiente conocimiento de la demanda & 7 \\
\hline & Falta de personalización en el servicio & 3 \\
\hline & Desconocimiento de las necesidades reales del nicho & 1 \\
\hline \multirow{3}{*}{ Normativas } & Carencia de normas que regulen el proceso & 1 \\
\hline & Obsolescencia en la norma aplicable & 1 \\
\hline & $\begin{array}{l}\text { Burocratismo en negocios de otras formas de la } \\
\text { economía }\end{array}$ & 1 \\
\hline \multirow{3}{*}{ Comercialización } & Insuficiente gestión de marketing & 7 \\
\hline & Insuficientes espacios & 6 \\
\hline & Oferta poco diversificada & 2 \\
\hline \multirow{3}{*}{ Economía Nacional } & Deficiente relación entre formas de la economía & 1 \\
\hline & Escasez de proveedores y materias primas & 1 \\
\hline & Diversificación de las actividades turísticas & 1 \\
\hline \multirow{3}{*}{ Empleo de Tecnologías } & Ausencia del comercio electrónico & 2 \\
\hline & Falta de aplicación de la TICs & 1 \\
\hline & $\begin{array}{l}\text { Empleo de formas de pago electrónico (Online y } \\
\text { Tarjeta) }\end{array}$ & 1 \\
\hline
\end{tabular}

Fuente: Elaboración propia.

Se señalan entonces, como parte del diagnóstico las principales oportunidades, amenazas, fortalezas y debilidades dirigidas a instalaciones que brinden servicios con productos de sobremesa cubanos y presten servicios al turismo.

Oportunidades $(\mathrm{O})$ :

O1: Mercado potencial seguro

O2: Incremento sostenido del arribo de visitantes al país. 
O3: Incremento a nivel mundial del interés por el turismo de compras.

O4: Aumento de los visitantes por viajes de cruceros a la isla.

O5: Existencia de pocos competidores.

O6: Interés de las autoridades locales por rescatar elementos de la cultura cubana para fomentar la actividad turística.

Amenazas (A):

A1: La marcada estacionalidad del turismo en Cuba.

A2: Cambios que sufre la política económica de Cuba.

A3: Cambios irregulares en el clima cubano.

A4: Conectividad a Internet.

Entre las principales fortalezas $(\mathrm{F})$ se pueden citar:

F1: Novedad del producto ofrecido en Cuba.

F2: Buenas prácticas en la gestión gastronómica.

F3: Conocimiento de los productos de sobremesa cubanos.

F4: Facilidades de establecer contratos con proveedores estatales.

F5: Posibilidad de establecer contratos con agencias de viajes y turoperadores nacionales e internacionales.

Además, se pueden mencionar debilidades (D) como:

D1: Asequibilidad del producto, pues resulta costoso para algunas personas.

D2: Aprovisionamiento de productos de sobremesa cubanos de alta gama.

A través de los análisis planteados se puntualizan criterios y propuestas para instalaciones que presten servicios al turismo, relativas al tema objeto de investigación:

Los aspectos principales en la gestión de la restauración y la sobremesa cubana, como son la tecnología, la preparación del personal, los materiales, la imagen del establecimiento y su ubicación en el mercado, deben tener un permanente monitoreo.

El maridaje juega un papel importante en el servicio de sobremesa. El conocimiento del mismo y sus características es de vital importancia para su gestión de venta. 
Los aspectos que a continuación se exponen, influyen de manera significativa en la gestión de venta del servicio de sobremesa cubana en un restaurante: preparación técnica del personal encargado de su venta, categoría de la instalación donde se venda, mercado que visite la instalación, tipo de productos característicos de este servicio que se venda. Tipo de comida que se oferte en el restaurante.

Propuesta de solución:

Los perfiles por competencias son relativos a puestos de trabajo que se desarrollan enfocados en la mejora necesaria del rendimiento de las instalaciones y de los recursos humanos que atienden procesos sustantivos en las mismas. De ahí que se relacionen categorías críticas de trabajo y competencias necesarias y suficientes para su diseño.

En la práctica regional, la construcción de perfiles por competencias ha sido fuente de solución a dificultades de índole similar, es el caso preciso de la comunidad iberoamericana cuando convino en estandarizar el proceso de elaboración de cócteles, solucionando los vacíos existentes en la construcción del perfil por competencias del cantinero o bartender.

Este sistema ha logrado presentar novedosas soluciones y crear organizaciones internacionales y gremiales de trabajo conjunto, favoreciendo esto la protección al consumidor correspondiente con la seguridad y calidad de productos y servicios y la estandarización de procesos.

El diseño del perfil por competencias del Habanosommelier, actor fundamental del proceso que se investigó, constituye una necesidad para la fortificación del modelo de protección a consumidores en instalaciones de restauración que presten servicios al turismo en La Habana. Atendiendo a la metodología presentada por Perelló Cabrera 2016, se propone una ficha técnica de la principal función del Habanosommelier.

Este diseño permite estructurar un procedimiento sólido de prestación del servicio de sobremesa, objeto de estudio, estandarizar el sistema de seguridad y calidad que se debe de prestar ante el consumo del propio servicio y evitar, por todos los medios, la contratación de personal no cualificado para atender este proceso. Es además un sólido compromiso al correcto proceder y atención de la administración a la problemática del intrusismo profesional en esta área, causante de las principales deficiencias identificadas.

\section{Ficha técnica del servicio de maridaje.}

\section{Objetivo.}

Trazar las pautas generales que garantizarán en la Cooperativa "Casa Haroca" el Servicio para grupos y clientes individuales consistente en la degustación de café, ron y tabaco, mediante la aplicación de lo dispuesto en el presente procedimiento de Trabajo. 


\section{Alcance.}

Aplicable al servicio al cliente de grupos e individuales desde su llegada a la instalación hasta la despedida final.

\section{Responsables.}

Son responsables cada uno de los trabajadores del salón, Capitán de Salón y Administrador.

\section{Definiciones.}

- Jefe de rango: es uno de los dependientes, que previamente ha sido designado por el jefe de brigada, para dar atención directa a las mesas de los clientes, en un área del salón, tiene subordinado uno o más dependientes para la prestación del Servicio y; asigna a uno de ellos, la función del suministro a esa área y las actividades relacionadas con las otras, y al otro dependiente, en caso de existir, le asigna funciones generales de apoyo.

- Habanosommelier: Es la persona encargada de impartir la clase magistral acerca del ritual del tabaco, así como de inducirlos en el maridaje de café, ron y habanos.

\section{Desarrollo.}

\section{Contratación y oferta.}

Se elaborará un contrato general cuyo objeto será la prestación de las ofertas y servicios de la Cooperativa "Casa Haroca" a las agencias receptivas nacionales. En este contrato se establecen las condiciones y obligaciones a cumplir por ambas partes relativo a los precios y formas de pago, las reservas y cancelaciones, las penalizaciones, la calidad de los servicios, la promoción y publicidad de las ofertas, las reclamaciones, las quejas, las compensaciones, la solución de divergencias, la vigencia y resolución del contrato, entre otros temas.

El proceso de negociaciones con los receptivos se efectuará centralmente por el Dpto. Comercial de la cooperativa, se firmarán los anexos específicos al contrato general, considerando las actividades específicas que oferte la Instalación a las Agencias.

Los contratos se firmarán por un año y anualmente se discutirán, se actualizarán o se renovarán en dependencia de los intereses de las partes.

\section{Durante el servicio:}

\section{Diagrama de flujo del proceso de atención de clientes de grupos:}


Figura 1. Diagrama de flujo del proceso

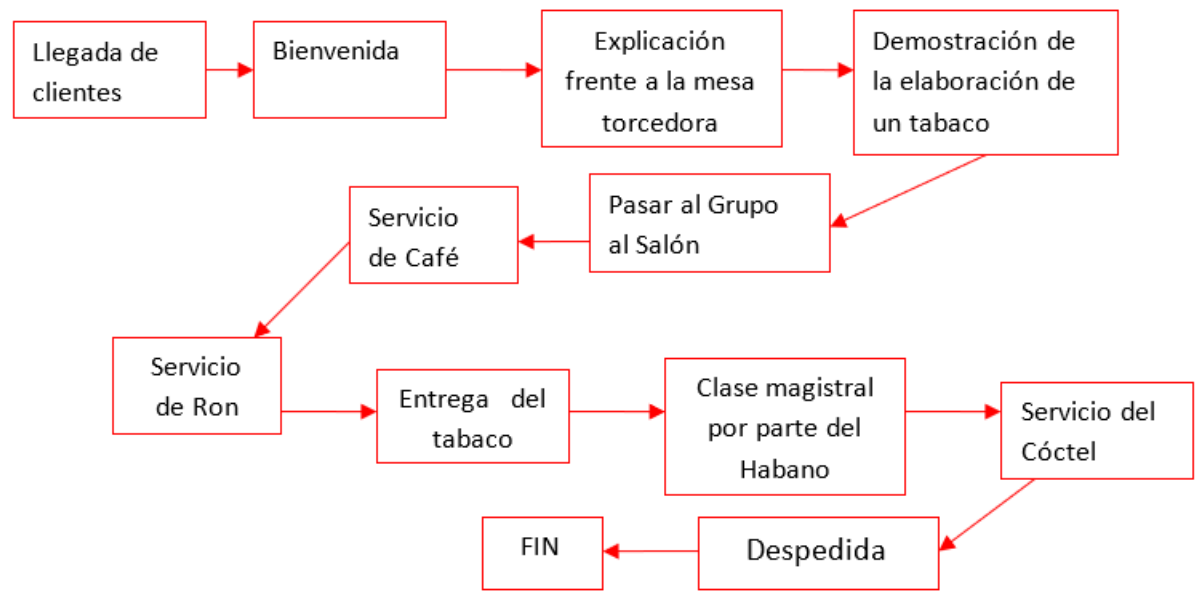

Fuente: Elaboración propia.

- Al arribar los clientes al establecimiento, se les dará una cordial bienvenida, el Capitán le dará la bienvenida con la frase siguiente: "Buenos días, tardes o noches" según corresponda al horario del día.

- El Habanosommelier se cerciorará, con el guía de la identidad del grupo y la cantidad de clientes reales. Indicándole que son esperados en el restaurante. y se comunica con el Capitán para darle la información.

- El Habanosommelier al recibir el aviso del Capitán de Salón, sobre la llegada del grupo, informará inmediatamente de ello a los dependientes y a la torcedora.

- El Habanosommelier los invita a pasar a la mesa torcedora para que reciban la información y demostración de cómo se elabora un Habano de forma artesanal, esta operación es realizada por una maestra torcedora.

- Al concluir esa actividad se les indica la posición a ocupar dentro del Salón para recibir el servicio de maridaje.

- Se procede a efectuar el servicio de café de acuerdo con lo establecido para este, inmediatamente se procede al servicio del ron, utilizándose la bebida

- Posteriormente se procede a la entrega y encendido del tabaco con la ayuda y demostración del Habanosommelier y finalmente se imparte la clase magistral por el mismo.

- En todo momento el personal de servicios estará atento a las mesas, solucionando cualquier solicitud, cambiando periódicamente los ceniceros, rellenando las copas de bebidas y retirando los insumos, botellas, etc. utilizados

- El Capitán de salón debe recoger la forma de pago (efectivo, voutcher, confirmación de pago, tarjetas de crédito) en la mesa o a la persona que viene a cargo del grupo.

- El Capitán de Salón, en caso de ser posible, acompañará a los clientes y al guía hasta la salida del salón para despedirlos.

- El Capitán de Salón entregará la propina al cajero si la hubiere.

- Se llevará a cabo la despedida, ayudando a los clientes a retirar sus asientos, conduciéndolos hasta la puerta e invitándolos a regresar. Se garantizará que las opiniones del grupo queden plasmadas en las Tarjetas o Libro del Cliente, la cual será 
presentada especialmente al guía o a los clientes que visiten la instalación individualmente.

\section{Control de la actividad.}

\section{Registros.}

Comanda u hoja donde se refleja en pedido.

\section{Ceremonia del Maridaje:}

Buenas (Tardes/Noches), Bienvenidos a la "Casa Haroca", mi nombre es (...) y estaré acompañándolos como su Habanosommelier en un viaje por los aromas de Cuba: café, tabaco y ron.

En este viaje serán testigos del maridaje de la sobremesa cubana, que no es más que la mezcla armónica de estos tres magníficos cultivos cubanos.

El café que estaremos degustando es el café Serrano, de fortaleza media y $100 \%$ arábico. Esta está entre las tres mejores marcas de Cuba, después del Cohíba y el Montecristo.

El Ron que usaremos es el Havana Club Añejo Especial, perteneciente a la gama de rones de Havana Club S.A. Este será mezclado con el Romeo y Julieta No. 2 de fortaleza media, que se encuentra entre las tres mejores marcas de Habanos de Cuba, después del Cohíba y el Montecristo, sucediéndole el Partagás, H. Upman y Hoyo de Monterrey.

Estas seis marcas son las más internacionalizadas y le dan el nombre de Habanos a los mejores tabacos de Cuba.

Breve Historia del Habano a utilizar. En este caso el Romeo y Julieta No.2:

En el caso del Romeo y Julieta, su fábrica nace en 1875, pero gana su máxima notoriedad en el año 1946 con la llegada del 1er ministro inglés Winston Churchill a La Habana. Cuando él prueba el Romeo y Julieta se enamora de él y dice: “(...) este es el mejor tabaco del mundo y yo lo quiero para mí (...)”. Es por ello que establece un contrato con la fábrica y determina que todos los meses se le envíe una edición especial solo para él. Cuando muere Churchill, la fábrica como estrategia de mercado nombra a su principal vitola pos su nombre.

Bien, pero vamos a explicarles cómo se fuma un buen Habano:

- El tabaco, a diferencia del cigarrillo, no se traga el humo, solo se mantiene en la boca y se expulsa.

- Es un filtro natural y si se divide en tercios solo se fuma hasta el último tercio (representado por la anilla) 
Esto que observan en mis manos se denomina cedrillo y es lo que vamos a utilizar para el encendido del Habano. El cedro, además de brindar aromas al habano, lo protege contara la humedad y las plagas de insectos.

Vamos ahora a servir el ron, y comienzo diciendo que el ron cubano posee 40 grados de alcohol, y en este caso su fortaleza va de ligera a media. En Cuba cuando abrimos una botella de ron los primeros en beber son los Orishas, por eso establecemos este pequeño ritual de pleitesía con el objetivo de pedir salud y prosperidad para nuestras familias.

Bien, primero vamos a olerlo, el ron a diferencia del vino no se agita, se mueve la nariz de derecha a izquierda y viceversa sobre el vaso. Lo primero que sentimos es su dulzor proveniente de la melaza de la caña de azúcar y con este sus notas a vainilla y vino seco. Vamos a probar solo un sorbo y lo pasaremos por toda la boca con el objetivo de adormecerla y prepararla para soportar las altas temperaturas del tabaco. ¡Brindemos por Salud!

Para encender el Habano encendemos el cedrillo y en un Angulo doble de 45 grados prendemos el Habano rotándolo por toda la llama de forma uniforme. Luego lo oxigenamos y está listo para fumar.

Existen tres formas de fumar un Habano:

- La simple

- La más famosa (Churchill, Chaplin, Fidel Castro, Che Guevara, Antonio Banderas)

- La elegante (mujeres)

Como una curiosidad:

- Churchill fumaba su Habano introduciendo la punta en su vaso de Whisky.

- Existe dentro del Festival del Habano, un evento denominado "El Cenicerazo" el cual consiste en ver quien lleva la ceniza del Habano más lejos. El récord lo tiene en la actualidad una mujer cubana que la llevó hasta $2 \mathrm{~mm}$ por debajo de la anilla.

\section{Conclusiones.}

- Los procedimientos, metodologías y pasos en la bibliografía disponible relativos a la gestión del producto servicio de sobremesa cubana en instalaciones que presten servicio al turismo en La Habana son insuficientes. Asimismo, se percibe una obsolescencia normativa con respecto al tema abordado.

- El resultado arrojado por el diagnóstico permitió establecer las prioridades sobre los aspectos relativos a la gestión del producto servicio de sobremesa cubana, entre las que sobresale el enfoque al cliente -partiendo del estudio de las necesidades reales del nicho- y la capacitación de los recursos humanos. Se evidencia la necesidad de enriquecer los insumos en cuanto a variedad e implementar el servicio de maridaje. 
- Las categorías detectadas sirvieron como fundamento en la elaboración de un procedimiento para atenuar el impacto negativo de los criterios identificados que deterioran el sistema de protección al consumidor en los servicios de sobremesa cubana. Esta herramienta garantiza la seguridad, objetividad y claridad en el proceso de la gestión de venta.

- Las principales variables que influyen en el deterioro del sistema de protección al consumidor en la gestión de productos de sobremesa cubana, identificadas a través de las herramientas y diagnósticos efectuados, son la comercialización, el enfoque al cliente y los recursos humanos. Asimismo, los principales criterios detectados fueron la poca diversificación de la oferta para maridaje, la no personalización del servicio y la insuficiente capacitación del personal.

\section{Referencias bibliográficas.}

AETi. (2016). ¿Dónde se ha encontrado el té más antiguo del mundo? Asociación Española de Té e Infusiones.

AHS. (2018). La cultura del café: Una tradición que nos marca más que el paladar. Asociación Hermanos Saíz.

Cerezal Mezquita, J. (2002). Los métodos científicos en las investigaciones pedagógicas. La Habana.

Colectivo de Autores. (2012). Gestión y evaluación de proyectos turísticos. La Habana: Félix Varela.

Consejo de Estado. (2012). Decreto No.309 Reglamento para las Cooperativas no Agropecuarias. Gaceta Oficial de la República. La Habana.

Consejo de Estado. (2012). Decreto-Ley No.305 de las Cooperativas no Agropecuarias. Gaceta oficial de la República de Cuba No.53. La Habana.

Consejo Regulador de la Denominación de Origen Protegido. (2017). El mundo del Habano. La Habana.

Controlaría General de la República. (2017). XI Comprobacion Nacional al Control Interno: funcionamiento de las Cooperativas no Agropecuarias. La Habana.

Cubaron S.A. (2016). El ron cubano no tiene secretos. Cubaron S.A., La Habana.

Danhke, G. (1986). Investigación y comunicación. Ciudad de México, México: Ed. McGrawHill de México.

EAEHT. (2016). Presentación de cursos para el año académico 2017. La Habana. 
Figueroa, V. (1996). El nuevo model agrario en Cuba bajo los marcos de la reforma económica. La Habana.

Martinez, S. (2018). Humidores Habana, mucho más que un slogan. Tiempo21.cu.

Morcillo, R. A. (2012). Procedimiento para la gestión de venta de servicios de sobremesa en restaurantes que presten servicio al turismo. Santa Clara.

OEC. (2016). Exportaciones, importaciones y socios comerciales. La Habana.

Oficina Económica y Comercial de España. (2016). Informe Económico y Comercial. La Habana.

OMT. (2020). La contribución de turismo a los objetivos de desarrollo sostenible en Iberoamérica. Organización Mundial del Turismo, Madrid. Obtenido de http://www.e-unwto.org/doi/book/10.18111/9789284420018

PCC. (2011). Lineamientos de la política esconómica y social del Partido y la Revolución. La Habana.

Piñeira, C. (2013). Cuba: Realidad y perspectivas del cooperativismo. La Habana, Cuba. Obtenido de tomado de www.centrocultural.cop/blogs

Quienes Somos. (21 de Abril de 2017). Recuperado el 13 de Junio de 2018, de Portal Corporación Cuba Ron SA: www.cubaron.com

RAE. (2019). Real Academia Española. Obtenido de Real Academia Española: http://dle.rae.es

Rivera, A., Labardor, O., \& Alfonso, J. L. (2012). Cooperativismo, gestión y desarrollo local. Santo Domingo, República Dominicana: Editora Corripio.

Rodríguez, J. L. (17 de marzo de 2018). CubaDebate. Recuperado el mayo de 2018, de http://www.cubadebate.cu

Tristá, G. (2013). Cooperativismo en Cuba: por elevar eficiencia y productividad. La Habana.

UNCTAD. (2016). Directrices de las Naciones Unidas para la Protección al Consumidor. Naciones Unidas, Nueva York y Ginebra.

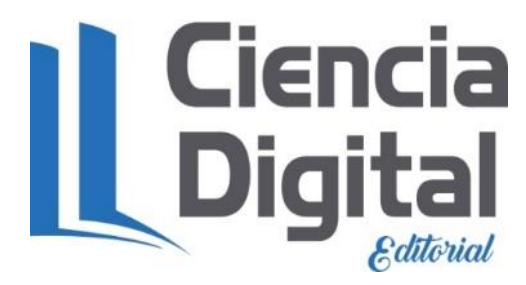


Para citar el artículo indexado

Milán Fonseca, P. P., González Basanta, O., \& Guerra Rodríguez, P. (2020). Variables de deterioro en la gestión de la sobremesa cubana y la protección al consumidor. AlfaPublicaciones, 2(1), 72-89. https://doi.org/10.33262/ap.v2i1.21

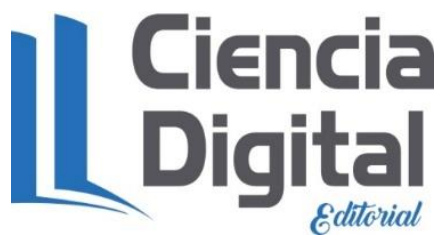

El artículo que se publica es de exclusiva responsabilidad de los autores y no necesariamente reflejan el pensamiento de la Revista Alpha Publicaciones.

El artículo queda en propiedad de la revista y, por tanto, su publicación parcial y/o total en otro medio tiene que ser autorizado por el director de la Revista Alpha Publicaciones.
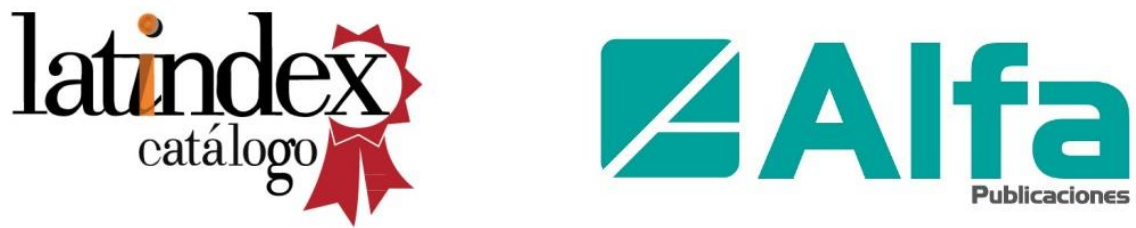